\title{
INVESTIGATION OF THE DYNAMIC BEHAVIOR OF MACHINE TOOLS DURING CUTTING BY OPERATIONAL MODAL ANALYSIS
}

\author{
J. Berthold ${ }^{1 *}$, M. Kolouch², J. Regel ${ }^{1}$, M. Putz ${ }^{1,2}$ \\ ${ }^{1}$ Chemnitz University of Technology, Professorship for Machine Tools and Forming Technology, Reichenhainer \\ Straße 70, 09126 Chemnitz, Germany \\ ${ }^{2}$ Fraunhofer Institute for Machine Tools and Forming Technology IWU, Reichenhainer Straße 88, 09126 Chemnitz, \\ Germany \\ *Corresponding author; e-mail: jan.berthold@mb.tu-chemnitz.de
}

\begin{abstract}
A common approach for the experimentally estimation of modal parameters is the Experimental Modal Analysis (EMA). EMA takes place in the standstill of the machine, consequently neglecting effects that only occur during the cutting process. Alternatively the Operational Modal Analysis (OMA) makes possible to investigate structures under operational loads and conditions. In this paper, a machine tool is investigated during cutting and the modal parameters are estimated by OMA, with several identification methods working in the frequency domain as well as in the time domain. These results are also contrasted to EMA to investigate the differences between cutting and standstill.
\end{abstract}

Keywords:

Machine tool; Dynamic; Operational modal analysis; Measurement

\section{INTRODUCTION}

The dynamic behavior of machine tool varies in the process phase, especially during cutting, compared to standstill due to process damping between tool and work-piece, gyroscopic moments of the spindle, especially at higher speeds, preloading of bearings and additional excitation sources by drives and moving components [Ozc15].

The standard instrument for dynamic investigations of machine tools is the Experimental Modal Analysis (EMA), which works in standstill of the machine by hammer or shaker excitation and therefore neglecting all the mentioned effects of the process state [Ewins 1986].

Nevertheless, the Operational Modal Analysis (OMA) makes possible to investigate structures especially under working conditions [Wan 2017]. This method is also used for analyzing the dynamic behavior of machine tools by excitation during cutting [Hui 2015]. Furthermore, there are existing approaches to excite the structure by traverse motion of components [Peng 2018]. However, this kind of excitation neglects damping effects occurring from cutting.

Within OMA, the modal parameters are identified only from response data without knowing the excitation. This results in many identification techniques that have been developed and modified in the history, to address special boundary conditions, e.g. unequal excitation levels, remaining harmonics, measurement noise, manual and automatic mode estimation [Masjedian 2009].

In investigations like [Mao 2016], [Li 2013], [Cai 2015], where OMA is used to identify modal parameters during cutting excitation, the techniques are used pragmatically without contrasting the results of several identification techniques or analysis setups.

This results in the question, in which way the several identification techniques of OMA address the issues of machine tools under operational conditions? To what extent to the characteristics of the OMA techniques like weighting factors, data processing or model order influence the results? Are there arising differences in the identified parameters, which could be put down to techniques itself?

\section{EXPERIMENTAL APROACH INVESTIGATED MACHINE TOOL}

\subsection{Approach}

Main concern is the comparison and evaluation of the results occurring from different identification techniques when performing OMA on machine tools during cutting. The techniques, which are considered in this paper, working on the one hand in the frequency domain, which are called Frequency Domain Decomposition (FDD) [Brincker 2001], and on the other hand, working in the time domain, which are called Stochastic Subspace identification (SSI) [Rainieri 2014]. For both domains, there are subgroups. In the frequency domain, there are the FDD, the Enhanced-FDD (EFDD) and the Curve-Fit-FDD (CFDD) [Jacobson 2008]. Regarding to the time domain, the SSI is subdivided in Unweighted Principal Components (UPC), Unweighted Principal Components with Merged Data Sets (UPCMerged), Principal Components (PC) and

Covariance Variate Analysis (CVA). All of these techniques are developed to deal with special boundary conditions during the measurements, regarding harmonic parts, 
measurement noise, unequal excitation level, nearby modes and so on. The excitation characteristics of a cutting process mostly include all of this issues.

To answer the question, which of these techniques with which analysis setup leads to the most suitable and plausible results, the identification is done within all these techniques and the modal parameters are evaluated by contrasting them to each other.

Subsequently, a preferred identification technique, dealing with the specifications of machine tools could be found.

In order to asses differences between the machine tool in cutting and standstill, the results of OMA are compared with the results of EMA.

\subsection{Machine tool and discretization}

All investigations are carried out on a three-axis machine tool. It consists of a fixed machine table, so that all movements are performed on the tool side. The workingspace could be described as a cuboidal block, in which the maximum traverse path is in the $\mathrm{X}_{\mathrm{NC}}$-direction with a length of $800 \mathrm{~mm}$. In $\mathrm{Y}_{\mathrm{NC}}$ - and $\mathrm{Z}_{\mathrm{NC}}$-direction $400 \mathrm{~mm}$ are available for each axis (see Fig. 1).
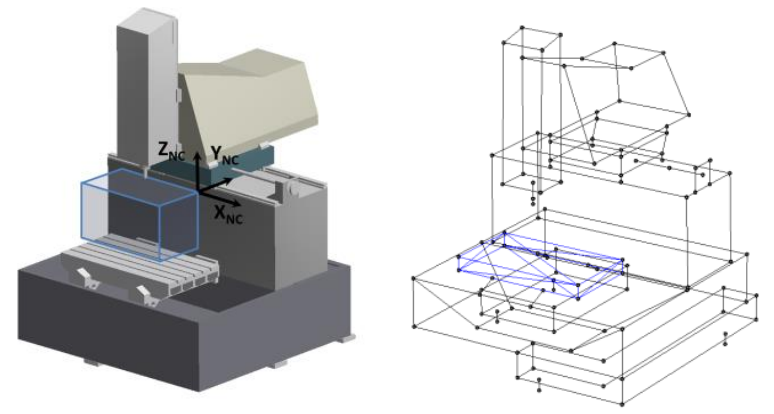

Fig. 1: CAD-model and wire frame model of the investigated machine tool.

The machine is discretised with 109 measurement points including machine bed and table, the base frame under the $\mathrm{X}$-slide and $\mathrm{X}$-slide itself as well as the $\mathrm{Y}$ - and Z-slide. Components which are inaccessible during cutting, like main spindle and tool changer, are neglected (see Fig. 1).

\subsection{Experimental Modal Analysis}

In order to get reference values for the comparison of standstill and cutting phase, an EMA is performed by relative excitation between machine table and a dummytool, mounted in the main spindle, with electromagneticshaker (see Fig. 2). This kind of excitation is chosen (instead of an impulse hammer) because the similar characteristics like a cutting process, to ensure comparability of the results.

The excitation takes place by a random-burst signal with a period of $2 \mathrm{~s}$ and with a force ratio of $50 \%$ (see Figure 5). In the frequency range the area around $300 \mathrm{~Hz}$ is conspicuous because of the higher amplitude. This results from the coupling of shaker to the machine tool and should be considered in case of comparison.

16 modes could be identified in the range from 10 to 400 $\mathrm{Hz}$ (see Tab. 1). The damping values starting at $3 \%$ at lower natural frequencies and decrease up to $0.15 \%$ at higher frequencies.

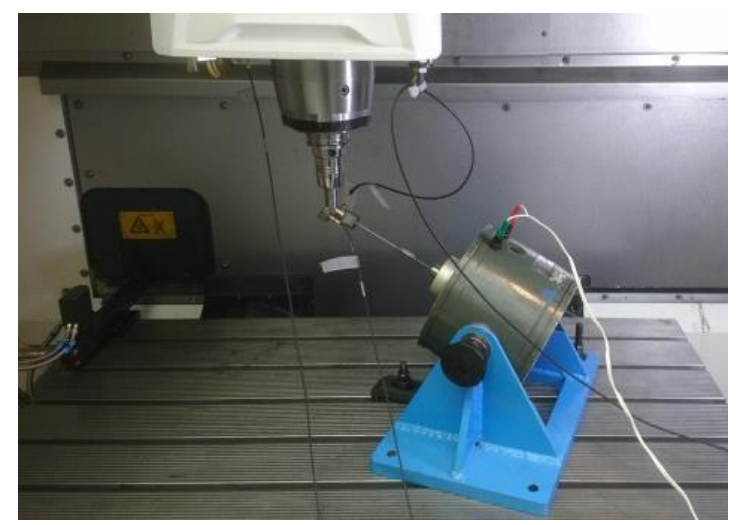

Fig. 2: Implementation of EMA with electromagneticalshaker.

Tab. 1: Modal Parameters identified by EMA in standstill.

\begin{tabular}{c|cc|c|cc}
\hline$\#$ & EF (Hz) & $\boldsymbol{\vartheta}(\%)$ & $\#$ & EF (Hz) & $\boldsymbol{\vartheta}(\%)$ \\
\hline 1 & 24.3 & 3.15 & 9 & 234.1 & 0.97 \\
2 & 52.1 & 1.59 & 10 & 260.1 & 0.70 \\
3 & 74.9 & 1.21 & 11 & 269.6 & 1.85 \\
4 & 87.4 & 3.35 & 12 & 283.3 & 0.76 \\
5 & 112.5 & 1.06 & 13 & 297.2 & 1.71 \\
6 & 137.9 & 1.86 & 14 & 356.7 & 0.19 \\
7 & 189.4 & 1.65 & 15 & 361.9 & 0.29 \\
8 & 211.2 & 0.15 & 16 & 392.3 & 0.67 \\
\hline
\end{tabular}

\section{IMPLENTATION OF OPERATIONAL MODAL ANALYSIS ON MACHINE TOOLS}

When performing OMA on machine tools during cutting some aspects have to be considered regarding to measurement, to ensure that the assumptions are met [Berthold 2017].

\subsection{Time-Invariance in Working-Space}

The identification of modal parameters with the FDD as well as the SSI techniques assume that the investigated structure can be considered as time-invariant. This assumption is in contrast to the working-space of machine tool. The dynamic behavior will change in dependency with every NC-position because of changing the relative position of the machine tool components to each other. Nevertheless, a cutting process requires exactly this relative movement because of the necessary cutting- and feed-motion.

To overcome this contradiction, it has been shown, that there are areas in the working-space in which these changes in the dynamic behavior are small and a timeinvariant dynamic behavior can be assumed [Putz 2016].

In Fig the FRF's along the $\mathrm{X}_{\mathrm{NC}}$-axis are depicted. It can be seen, that there are very small changes in the FRF's over the $\mathrm{X}_{\mathrm{NC}}$ position. So the motion along this axis when considering a cutting process should make possible an identification with plausible modal parameters. In [Berthold 2018] the dynamic behavior of the machine tool over the whole working-space is described in detail. 


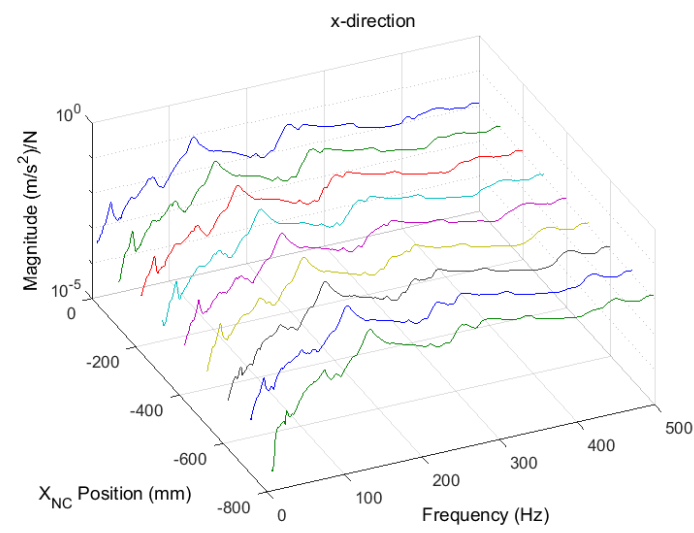

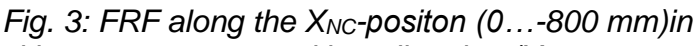
working-space measured in $x$-direction $\left(Y_{N C}=-200 \mathrm{~mm}\right.$ and $Z_{N C}=-300 \mathrm{~mm}$ are constant).

\subsection{Excitation by cutting process}

Furthermore attention has to be paid to the cutting process which is used when performing OMA. To generate a process, which has the characteristics of White noise and that is free of harmonics, the cutting parameters have to be modulated during cutting.

In the described example (see Fig. 3), this is done by a milling process with random spindle speed values, changing along the traverse path in the $\mathrm{X}_{\mathrm{NC}}$-axis. The values change in the range from 600 to $1400 \mathrm{~min}^{-1}$ every millimeter of the traverse path for a workpiece with the length of $300 \mathrm{~mm}$. In this spindle speed range, the actual values following the commanded values with time delay but without collapse of the feed, by adjusting the feed hold value parameter in the control system. Other cutting parameters such as feed rate $v_{f}=300 \mathrm{~mm} / \mathrm{min}$, immersion $\mathrm{a}_{\mathrm{e}}=66 \%$ (tool-diameter $\mathrm{d}_{\text {tool }}=40 \mathrm{~mm}, 4$ cutting edges with equal pitch angle) and depth of cut $a_{p}=1 \mathrm{~mm}$ are kept constant. This leads to a kind of random modulation of the chip-thickness along the motion. This excitation concept and also other concepts of a broadband excitation by cutting forces are discussed more in detail in [Berthold 2016].

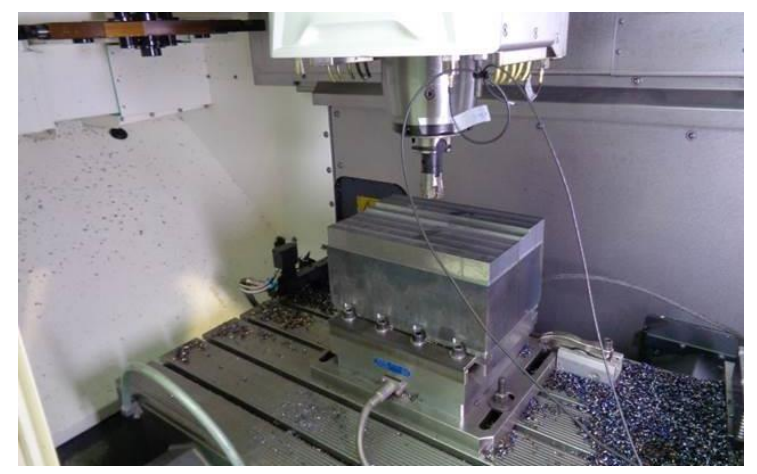

Fig. 3: Workpiece mounted on Kistler dynamometer for excitation by cutting process.

The resulting cutting force could be seen in time and frequency domain in Fig. 4 (also in comparison with the shaker excitation by EMA). The level of the Magnitude of the OMA is in a wide frequency range nearly the same as for EMA. However, the excitation by the cutting process seems to be more powerful in the lower frequency range (up to $100 \mathrm{~Hz}$ ), characterized by magnitudes that are three times higher than in the case of EMA. This should be kept in mind, considering the subsequently identification. An area of one remaining harmonic could only be seen in the near of $5 \mathrm{~Hz}$, which results from the spindle speed modulation period. Nevertheless, the first eigenfrequencies should appear above this value, it also should be noted in the subsequently identification.
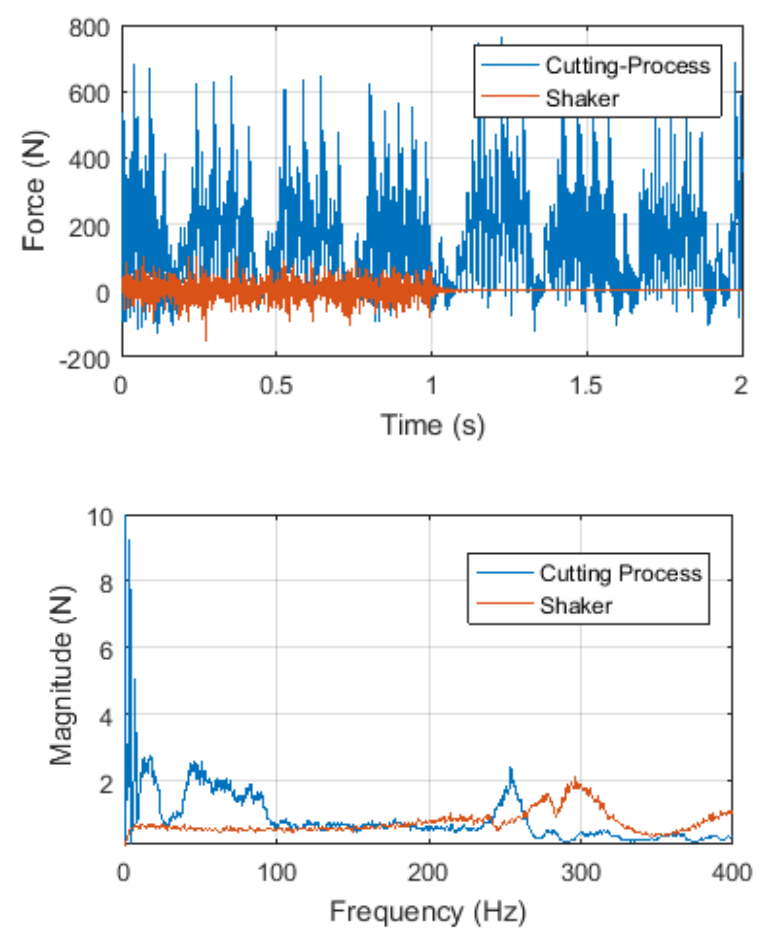

Fig. 4: Excitation force by cutting process and shaker excitation (above: time domain, below: frequency domain).

\subsection{Measurement Setup}

The wire frame model of the machine (see Fig. 1) depicts the positon of the measurement points, in which the response to the milling process is recorded with acceleration sensors. In consequence of inaccessibility and moving parts, not at all points a response is measured.

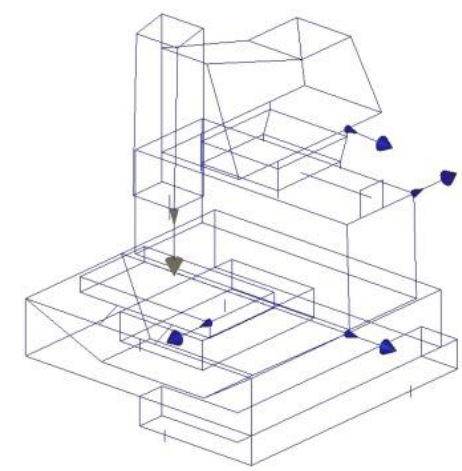

Fig. 5: Reference sensors (arrows) on machine structure.

The measurement is divided into 44 sequences, which also means the same number of milling paths. Additionally to EMA, OMA requires the definition of reference sensors for scaling and merging the recorded data of different sequences into one result. The Sensors are located at the machine bed, machine table, the base frame under the $X$ slide and the Y-slide (see Fig. 5). The used measurement equipment allows only 2 three-axial-acceleration sensors as roving sensors in every sequence. 


\section{IDENTIFICATION OF MODAL PARAMETERS BY OPERATIONAL MODAL ANALYSIS}

In order to get an overview of the measured data and to evaluate the measured response signals, the singular value decomposition (SVD) is performed [Herlufsen 2005].

In Fig. 6 the five calculated singular values are depicted, describing the most relevant information. It could be seen by looking at the first singular value, that lower frequency range, 0 to $25 \mathrm{~Hz}$, is characterized by many high peaks, probably resulting from the process, which will make an exact identification of modes difficult in this area. Besides this, the dynamic range is about $30 \mathrm{~dB}$, which could be interpreted as an adequate excitation of the structure by the modified milling process. In the rest of the frequency range, there are no remaining harmonics but some interesting peaks, which probably could be identified as natural frequencies. Especially the areas from 70 to $100 \mathrm{~Hz}$, around $135 \mathrm{~Hz}$, from 180 to $200 \mathrm{~Hz}$ and from 230 to $340 \mathrm{~Hz}$ look promising.

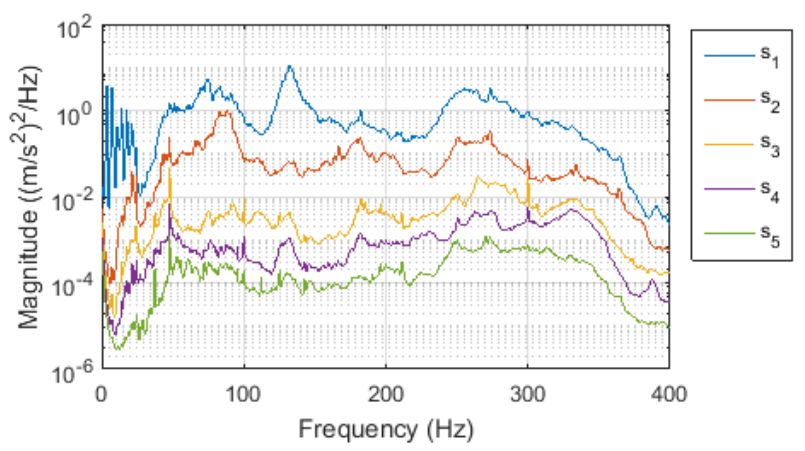

Fig. 6: Singular Value Decomposition of the response signals measured during milling.

\subsection{Frequency Domain}

Modal parameters are identified for the same response data but with different techniques (FDD, EFDD, CFDD). Starting from FDD with 11 modes, the number of modes increases to 15 for EFDD and has its maximum with 16 modes found by CFDD (see Tab. 2). Similar frequencies are assigned in the table in the same line to each other. The most modes are found by CFFD, including also the modes identified by the other techniques. To the first frequency at $3.8 \mathrm{~Hz}$ belongs an extremely high damping with $72 \%$, which is probably no eigenfrequency but resulting from the excitation and spindle speed modulation frequency.

The first plausible eigenfrequency is found by $51.6 \mathrm{~Hz}$ with $2.9 \%$ damping rate. Compared to the results of EMA, the mode at $24 \mathrm{~Hz}$ is not found by OMA.

Except for the mode at $300 \mathrm{~Hz}$, the results of EFDD and CFFD are nearly the same, considering the frequency values. The damping values are lower in the CFDD (over $180 \mathrm{~Hz}$ in the range from 0.2 to $0.6 \%$ ), also lower than in EMA, which is in contrast to the presumption, that the process damping leads to higher values.

When comparing the mode shapes of the results by the MAC [Allemang 2003], there occurring some similarities but also differences, although the differences in techniques is not that different. Especially EFDD and CFDD differ in mode shapes, 6 out of 15 mode shape parings have a MAC higher $80 \%$. In conclusion, with the CFDD the most eigenfrequencies could be identified and even if the damping values are smaller than the presumption expects, in general, the results of this technique can be seen to be most plausible.

Tab. 2: Modal Parameters identified by FDD-Techniques.

\begin{tabular}{cc|ccc|ccc}
\hline FDD & & EFDD & & & \multicolumn{3}{|l}{ CFDD } \\
$\#$ & EF (Hz) & $\#$ & $E F(H z)$ & $\vartheta(\%)$ & $\#$ & EF (Hz) & $\vartheta(\%)$ \\
\hline 1 & 3.6 & 1 & 3.5 & 8.54 & 1 & 3.8 & 71.66 \\
& & 2 & 51.9 & 6.45 & 2 & 51.6 & 2.85 \\
2 & 66.4 & 3 & 74.2 & 4.37 & 3 & 74.4 & 2.16 \\
3 & 83.6 & 4 & 89.3 & 0.87 & 4 & 92.7 & 0.53 \\
4 & 132 & 5 & 131.9 & 3.22 & 5 & 132.5 & 2.30 \\
& & 6 & 183.7 & 0.86 & 6 & 183.6 & 0.47 \\
5 & 182 & 7 & 196.6 & 0.54 & 7 & 195.1 & 0.53 \\
6 & 211.6 & 8 & 213.2 & 0.88 & 8 & 210.1 & 0.53 \\
7 & 254.8 & 9 & 256.1 & 0.91 & 9 & 256.5 & 0.57 \\
8 & 274.8 & 10 & 273.8 & 0.78 & 10 & 273.7 & 0.45 \\
& & & & & 11 & 286.6 & 0.25 \\
9 & 306 & 11 & 301.9 & 0.71 & 12 & 299.0 & 0.18 \\
& & 12 & 324.0 & 0.00 & 13 & 320.2 & 0.23 \\
& & 13 & 356.1 & 0.27 & 14 & 361.6 & 0.39 \\
10 & 365.2 & 14 & 361.0 & 0.92 & 15 & 365.2 & 0.16 \\
11 & 391.2 & 15 & 389.9 & 0.31 & 16 & 390.0 & 0.29 \\
\hline
\end{tabular}



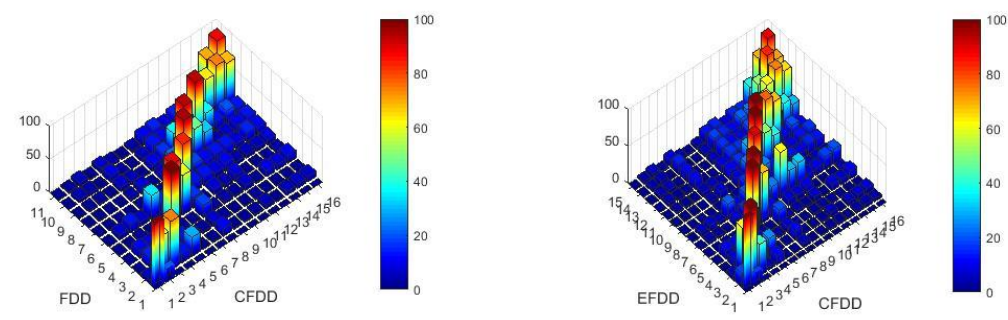

Fig. 7: Comparison of the mode shapes of FDD-techniques by MAC.

The benefit of CFFD lies in the fact, that the identification is done directly in the frequency domain by curve-fitting a polynomial description of the positive half-power spectrum. This is in opposite to the EFFD, where the transformation in the time domain can lead to bias errors in the damping values, because only a part of the signal is transformed [Jacobson 2008]. Also, EFFD assumes clearly separated modes. FDD could be used just to get a quick over of the investigated structure.

\subsection{Time Domain}

In Table 3 the modal parameters are shown, that are identified by the four techniques UPC Merged Data Sets, UPC, PC and CVA.

Again, the same identified frequencies are assigned to each other in the table. Like in all the FDD-techniques, in reference to EMA, no mode is identified at $25 \mathrm{~Hz}$. The first mode is found at about $47 \mathrm{~Hz}$.

Summarizing, the highest number of modes could be identified by the CVA technique, whereby the other techniques also result in further plausible modes, which are not found by CVA. Especially the UPC Merged Data Sets technique with plausible modes at $92.8,186.3$ and $237.6 \mathrm{~Hz}$ should be pointed out. The advantage of this technique consists in the fact, that all measurement sequences are treated together as one data set and so the parameters are identified by considering all information at one time, which surly leads to plausible results when evaluating eigenfrequencies. This is in contrast to the other SSI techniques, in which the modal parameters are separately identified for each sequence and combined afterwards. For every sequence a stable eigenfrequency has to be identified, in order to obtain an eigenfreqency of the whole machine tool. This could lead to non-recognized eigenfrequencies, especially when they are not excited equally. The advantage of the CVA lies in the fact, that for every sequence the stability criteria's (differences in frequency, damping and mode shapes) could be adjusted separately and so especially the problem of unequal excitation and closed modes could be processed much more focused.

Generally, higher damping values are found in comparison with the FDD-techniques as well as with EMA. But especially the damping values of about $20 \%$ (found with UPC Merged Data Sets), seem to be overestimated. Here the CVA technique leads to the most plausible results with values in the range from 9.8 to $1.6 \%$, which is consistent to the presumptions.

When looking at the mode shapes, the modal parameters of the CVA are used as a reference and the results of the other techniques are compared by using the MAC (see Fig. 8). In contrast to the FDD-techniques the mode shapes vary even more. Just a few similarities could be found. High MAC values especially exist at about (depending on the technique) 72 and $132 \mathrm{~Hz}$. These mode shapes are very distinctive, characterized by clear motions of single machine components, e.g. yawing of the $\mathrm{Y}$-slide around the $Z_{\mathrm{NC}}$-axis in the opposing cycle to the machine bed (at 72 $\mathrm{Hz}$ ) or on the other hand rolling of the $\mathrm{Y}$-slide around the $Y_{N C}$-axis (at $132 \mathrm{~Hz}$ ). The differences in the mode shapes mostly result from non-plausible overshooting of the mode shape vectors of a few measurement points. This depends on the used technique. It has to be mentioned, that the difference in the techniques is the weighting of the response data because there is no information about the excitation of the structure in contrast when performing EMA. In this context, the strongest influence on the response data is practiced by the CVA technique [Hermans 1999, Rainieri 2014]. The UPC techniques does not use weighting and PC only sparsely. Regarding to the excitation by modified cutting process, the excitation level is not equal over the whole frequency range at all modes. So the use of the CVA technique appears to be most reasonable, because it deals with unequal excitation, the eigenfrequencies and damping values are reasonable and the technique allows to adjust the identification process for every measurement sequence individually.
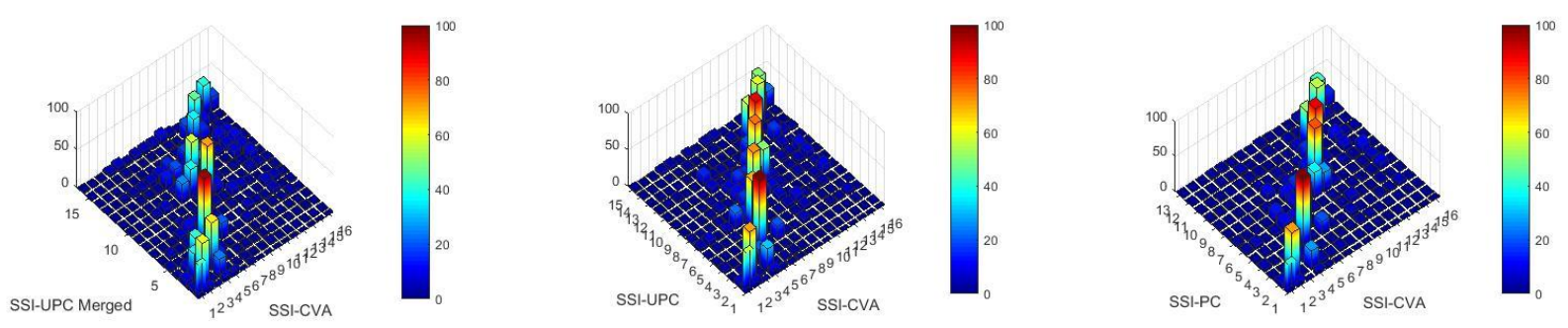

Fig. 8: Comparison of the mode shapes of SSI-techniques by MAC. 
Tab. 3: Modal Parameters identified by SSI-Techniques.

\begin{tabular}{|c|c|c|c|c|c|c|c|c|c|c|c|}
\hline \multicolumn{3}{|c|}{ UPC Merged Data Sets } & \multicolumn{3}{|l|}{ UPC } & \multicolumn{3}{|l|}{ PC } & \multicolumn{3}{|c|}{ CVA } \\
\hline$\#$ & $\mathrm{EF}(\mathrm{Hz})$ & $\vartheta(\%)$ & $\#$ & $\mathrm{EF}(\mathrm{Hz})$ & $\vartheta(\%)$ & $\#$ & $\mathrm{EF}(\mathrm{Hz})$ & $\vartheta(\%)$ & $\#$ & $\mathrm{EF}(\mathrm{Hz})$ & $\vartheta(\%)$ \\
\hline 1 & 43.9 & 19.68 & 1 & 47.3 & 10.07 & 1 & 47.1 & 11.77 & 1 & 47.3 & 7.05 \\
\hline \multirow[t]{2}{*}{2} & 72.0 & 13.78 & 2 & 72.8 & 8.35 & 2 & 72.9 & 7.43 & 2 & 72.4 & 3.91 \\
\hline & & & & & & & & & 3 & 83.93 & 9.84 \\
\hline 3 & 86.6 & 10.07 & 3 & 86.1 & 6.84 & 3 & 86.4 & 7.19 & 4 & 89.2 & 6.22 \\
\hline 4 & 92.8 & 19.97 & & & & & & & & & \\
\hline \multirow[t]{2}{*}{5} & 131.3 & 3.94 & 4 & 131.8 & 2.91 & 4 & 131.7 & 2.97 & 5 & 132.8 & 2.64 \\
\hline & & & 5 & 179.6 & 6.59 & 5 & 183.2 & 5.97 & 6 & 182.4 & 3.21 \\
\hline 6 & 186.3 & 10.08 & 6 & 186.1 & 5.65 & & & & & & \\
\hline 7 & 201.8 & 14.98 & 7 & 204.1 & 6.98 & 6 & 205.7 & 7.78 & 7 & 210.5 & 3.25 \\
\hline 8 & 237.6 & 18.43 & & & & & & & & & \\
\hline 9 & 254.8 & 2.84 & 8 & 248.2 & 3.33 & & & & 8 & 250.1 & 2.52 \\
\hline 10 & 256.0 & 5.39 & & & & 7 & 256.0 & 2.48 & 9 & 254.4 & 2.09 \\
\hline 11 & 257.0 & 3.19 & 9 & 257.2 & 2.62 & & & & & & \\
\hline 12 & 265.4 & 3.71 & 10 & 262.6 & 2.45 & 8 & 263.7 & 2.14 & 10 & 262.0 & 2.19 \\
\hline \multirow[t]{2}{*}{13} & 270.1 & 9.29 & 11 & 273.2 & 1.87 & 9 & 273.6 & 2.07 & 11 & 273.79 & 1.55 \\
\hline & & & 12 & 286.2 & 3.40 & 10 & 286.28 & 3.27 & & & \\
\hline 14 & 297.8 & 8.96 & 13 & 299.5 & 4.04 & 11 & 299.64 & 2.59 & 12 & 301.38 & 2.16 \\
\hline 15 & 315.4 & 7.49 & & & & & & & 13 & 315.2 & 2.38 \\
\hline \multirow[t]{3}{*}{16} & 338.7 & 12.14 & 14 & 331.1 & 3.24 & 12 & 329.1 & 4.33 & 14 & 332.8 & 2.75 \\
\hline & & & 15 & 353.6 & 3.19 & 13 & 349.2 & 3.82 & 15 & 351.31 & 2.72 \\
\hline & & & & & & & & & 16 & 368 & 2.04 \\
\hline
\end{tabular}

\section{DISCUSSION OF THE RESULTS}

\subsection{Comparison of the OMA techniques}

The different identification techniques, working in frequency and time domain, result in modal parameters with similarities but also differences. Because the CFDD and the SSI-CVA lead to the most plausible results of the particular domains, they are compared and discussed more in detail (see Fig. 9).

Regarding to the eigenfrequencies, it is interesting, that the variations are very small. On the other hand, there are eigenfrequencies, which could be only identified by one technique, e.g with CFDD at 195, 287 and $390 \mathrm{~Hz}$ or in contrast with SSI-CVA at 84, 250, 262 and $333 \mathrm{~Hz}$, whereby every identified mode in each case seems to be plausible considering the results of EMA.

When looking at the damping values, the SSI-CVA leads to appreciably higher values than the CFDD does, partly ten times higher. A reason could be the overestimation with to high model order in the case of using SSI. Mostly, when identifying the modal parameter by looking at the stability charts of each sequence of the measurement, the chosen model order is higher than needed to ensure, that every possible mode is taken into account and no one is neglected. The higher the chosen order, the higher the chance that modes will get stable. Afterwards, when determining the modal parameters from the state space matrices, the damping could be overestimated.

Considering the modes shapes, there are only two similarities at 132 and $273 \mathrm{~Hz}$. The differences are mostly occurring from oscillation with antiphase or with higher amplitude than neighbour points.

In conclusion, the results of the SSI-CVA seem to be more reasonable, because as assumed the damping values are increasing in process and also the related mode shapes appear comprehensible. Furthermore, the weighting of the input data to identify also weakly excited modes, which is done in SSI-CVA, seems to be appropriate, when looking at the cutting force spectra.

\subsection{Comparison of OMA and EMA}

Because the results of the SSI-CVA are evaluated as the most reasonable regarding all OMA-techniques, these identified modal parameters are compered to results of EMA (see Fig. 10).

As it has been mentioned before, the first mode of EMA at $25 \mathrm{~Hz}$ could not be identified by OMA. Generally, the eigenfrequencies mostly decrease under process conditions, the maximum values is $-9.3 \%$ compared to standstill (from $52.1 \mathrm{~Hz}$ to $47.3 \mathrm{~Hz}$ ) on average it is about $-2.2 \%$. In general, this changes are very small and could be described by changing boundary conditions between standstill and cutting. This could result because of friction by the motion of the components, change of preloads and clearance but also by changing NC-position and accompanying with small changes in the dynamic behaviour.

In contrast, all the damping values increasing under process conditions, the changes are enormous with values over 20 times higher than in standstill, e.g. from $0.15 \%$ at $211.2 \mathrm{~Hz}$ to $3.25 \%$ at $210.5 \mathrm{~Hz}$.

This increase could be explained on the one hand by process damping resulting from the contact conditions of tool and work-piece during milling. On the other by changing contact conditions in bearings and guide ways. The gyroscopic moments which would also explain changes in damping, stiffness and mode shapes [Özsahin 2011] should be effective probably in higher frequency ranges which are not investigated. 

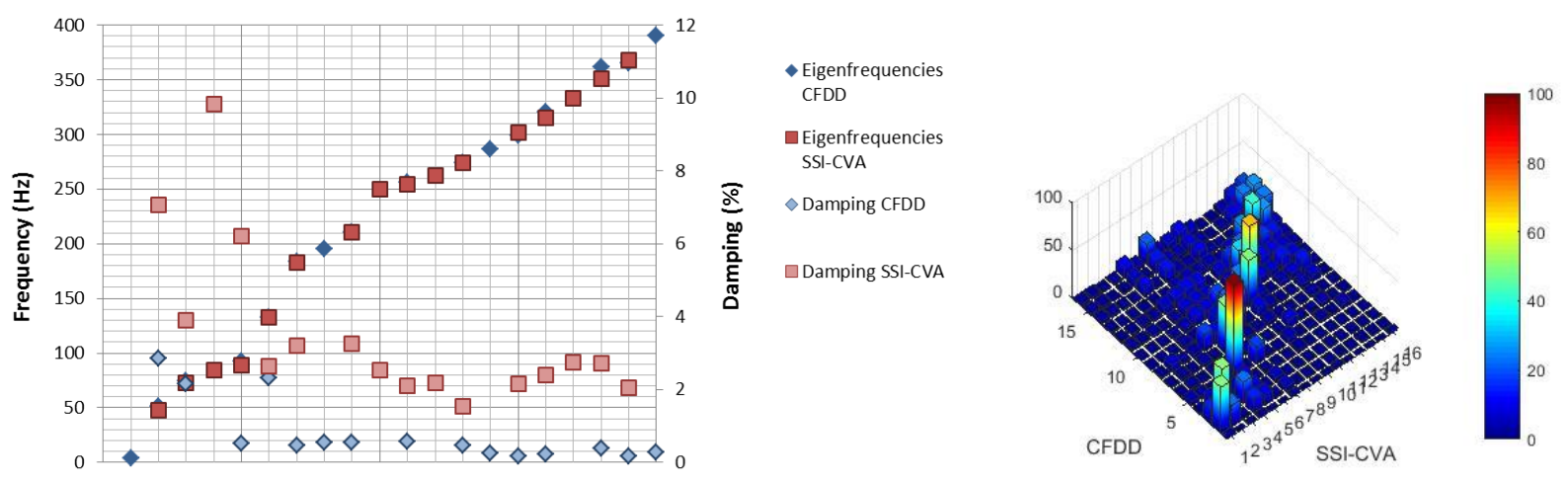

Fig. 9: Eigenfrequencies, Damping and MAC values identified by OMA techniques in frequency and time domain.
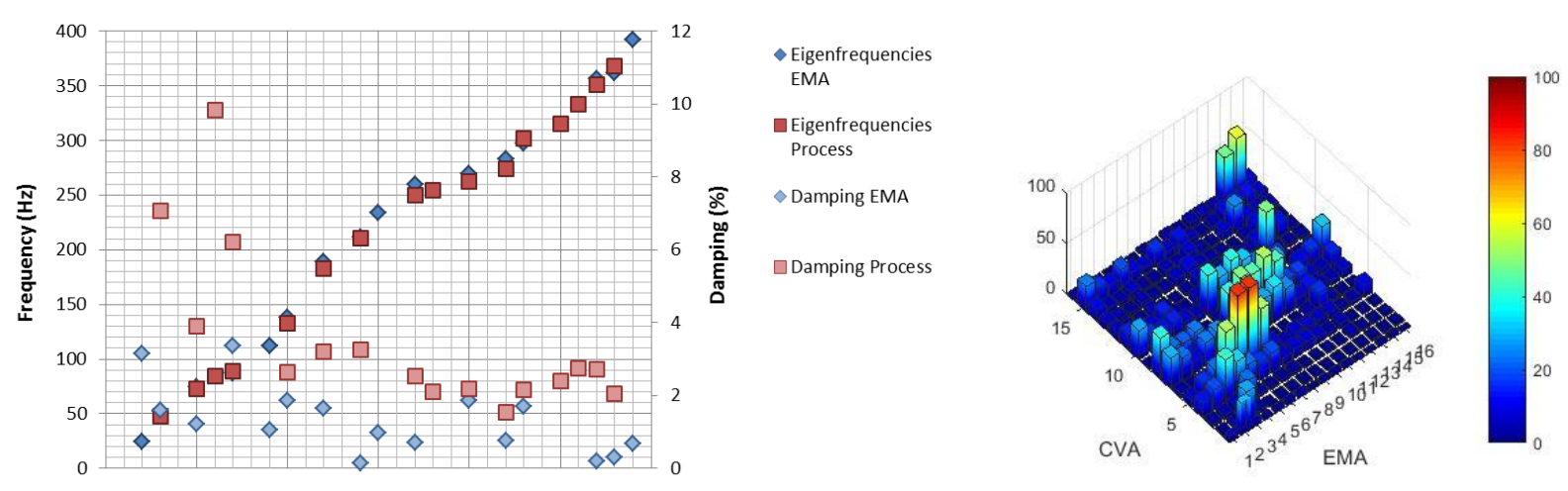

Fig. 10: Eigenfrequencies, Damping and MAC values identified by EMA in standstill and OMA during process.

Furthermore, changes in mode shapes are obviously. Considering the MAC values, there are only appreciable similarities at $132 \mathrm{~Hz}$. Otherwise, the mode shapes identified under process conditions seem to have more relative motion between the components, e.g. the relative movements at contact points, e.g. between guideway and guide-shoes seem to be higher in the process case. This could probably be explained by the different kind of excitation. The shaker excitation of EMA only works locally, while the process excitation, probably by the excitation in other locations, e.g. the main spindle, the drives and the vibrations caused by the motion of the components.

It should be mentioned, that the mode shapes of OMA are not scaled to the excitation in contrast to EMA, which should be kept in mind, especially when comparing mode shapes.

\section{CONCLUSION AND OUTLOOK}

The dynamic behavior of a machine tool is investigated in the standstill by EMA and under process conditions, during a modified milling process, by OMA.

The modal parameters are identified from the response measurement during cutting by three frequency domain techniques and four time domain techniques. The results of each domain are contrasted among each other. The CFDD results in the most reasonable modal parameters in frequency domain, whereby the results of the three techniques are very similar. In the time domain every techniques delivers slightly different modal parameters.
The results of the SSI-CVA could be seen as the most plausible considering damping values and mode shapes. The reason probably lies in the weighting of the response data, which makes possible to deal with an unequal excitation level.

Comparing the results of EMA in standstill and OMA during milling, it can be seen, that the eigenfrequencies decreasing sparsely and the damping increases during cutting. Furthermore the mode shapes are changing. The excitation of the milling seems to be more concerning the whole machine structure. So, OMA delivers the possibility to investigate the dynamic behavior during the cutting process and makes possible the tailored reaction to weak points of the machine.

Further studies will address investigations about the scaling of mode shapes identified by OMA as well as the use of OMA for structure health monitoring.

\section{REFERENCES}

[Allemang 2003] Allemang, R. J. The modal assurance criterion - twenty years of use and abuse., Sound and vibration, 2003, 37, pp. 14-23

[Berthold 2016] Berthold, J. and Kolouch, M. and Wittstock, V. and Putz, M. Broadband excitation of machine tools by cutting forces for performing operational modal analysis. MM Science Journal, 2016, pp. 1473-1481

[Berthold 2017] Berthold, J. and Kolouch, M. and Wittstock, V. and Putz, M. Aspects of the investigation of the dynamic 
behaviour of machine tools by operational modal analysis. MM Science Journal, 2017, 5, pp. 2013-2019. doi:10.17973/mmsj.2017_12_201777

[Berthold 2018] Berthold, J. and Kolouch, M. and Wittstock, V. and Putz, M. Identification of modal parameters of machine tools during cutting by operational modal analysis. Procedia CIRP 2018, 77, pp. 473 - 476. ISSN 2212-8271. 8th CIRP Conference on High Performance Cutting (HPC 2018)

[Brincker 2001] Brincker, R. and Zhang, L. and Andersen, P. Modal identification of output-only systems using frequency domain decomposition. Smart materials and structures, 2001, Vol.10, No.3, pp. 441-445

[Cai 2015] Cai, H. and Luo, B. and Mao, X. and Gui, L. Song, B. and Li, B. and Peng, F. A Method for Identification of Machine-tool Dynamics under Machining, Procedia CIRP, 2015, 31, pp. 502-507.

[Hui 2015] Hui, C. and Xinyong, M. and Bin, L. and Bo, L. Estimation of FRFs of machine tools in output-only modal analysis. The International Journal of Advanced Manufacturing Technology, 2015, 77, 1-4, pp. 117-130. ISSN 0268-3768. doi: 10.1007/s00170-014-6439-6

[Ewins 1986] Ewins, D. J. Modal testing: Theory and Practice. Letchworth, Hertfordshire, England: Research Studies Press Ltd., 1986

[Herlufsen 2005] Herlufsen, H. and Andersen, P. and Gade, S. N. and Möller, N. Identification techniques for operational modal analysis - an overview and practical experiences. Proceedings 1st IOMAC Conference, 2005.

[Hermans 1999] Hermans, L. and Van der Auweraer, $H$. Modal testing and analysis of structures under operational conditions: industrial applications. Mechanical Systems and Signal Processing, 1999, 13, pp. 193-216

[Jacobson 2008] Jacobsen, N.-J. and Andersen, P. and Brincker, R. Applications of Frequency Domain Curve-fitting in EFDD Technique, In: Conference Proceedings : IMACXXVI : a Conference \& Exposition on Structural Dynamics, Orlando, Florida, February 4-7, 2008.

[Li 2013] Li, B. and Hui, C. and Xinyong, M. and Junbin, H. and Bo, L. Estimation of CNC machine-tool dynamic parameters based on random cutting excitation trough operational modal analysis, International Journal of Machine Tools and Manufacture, 2013, 71, pp. 26-40.

[Mao 2016] Mao, X. and Yan, R. and Cai, H. and Li, B. and Luo, B. and $\mathrm{He}, \mathrm{S}$. A complete methodology for identifying dynamics of heavy machine tool through operational modal analysis, Proceedings of the Institution of Mechanical Engineers, Part B: Journal of Engineering Manufacture, 2016, 230, pp.1406-1416.

[Masjedian 2009] Masjedian, M. H.and Keshmiri, M. A review on operational modal analysis researches: classification of methods and applications. IOMAC09 - 3rd International Operational Modal Analysis Conference, 2009, pp. 707-718

[Özsahin 2011] Özsahin, O. and Budak, E. and Özgüven, $\mathrm{H}$. N. Investigating Dynamics of Machine Tool Spindles under Operational Conditions. Advanced Materials Research, 2011, 223, pp. 610-621

[Peng 2018] Peng, Y. and Li, B. and Mao, X. and Liu, H. and Qin, C. and He, H. A method to obtain the inprocess FRF of a machine tool based on operational modal analysis and experiment modal analysis. The International Journal of Advanced Manufacturing Technology 2018, 95, 2018 9, pp. 3599-3607. ISSN 1433-3015. doi:10.1007/s00170017-1405-8

[Putz 2016] Putz, M. and Wittstock, V. and Kolouch, M. and Berthold, J. Investigation of the Time-invariance and Causality of a Machine Tool for Performing Operational Modal Analysis. Procedia CIRP, 2016, 46 pp. 400 - 403. ISSN 2212-8271. 7th HPC 2016 -CIRP Conference on High Performance Cutting

[Rainieri 2014] Rainieri, C. and Fabbrocino, G. Operational modal analysis of civil engineering structures, Springer Verlag New York Heidelberg Dordrecht London, 2014

[Wan 2017] Wan, M. and Feng. J. and Ma, Y.C. and Zhang, W.H.. Identification of milling process damping using operational modal analysis, International Journal of Machine Tools and Manufacture, 2017, 122, pp. 120-131. 